emission was assumed, and a universal 1-nsec lifetime was taken to allow us to estimate the fraction of the metastable emitters which decayed within the distance viewed by our $\mathrm{x}$-ray detector. We thus have clear and direct experimental evidence that both two- and three-electron chlorine systems are produced which have lifetimes in the nanosecond region and which de-excite at least partially by $K \alpha$ x-ray emission. We further find that an appreciable fraction (about $60 \%$ ) of our metastable $x$-ray emitters belong to systems of more than three electrons. The number of $x$ rays detected from these systems is large because of the charge-state equilibrium at $45 \mathrm{MeV}$, in spite of the small probability per emerging ion for their production. The appropriate experiment to detect these species directly in a coincidence experiment is in preparation.

The identification of the charge states of these $x$-ray emitters in no way settles the question of their identity. Sellin et $a l .^{1}$ have attributed their metastable Auger-electron emitters to quartet states in three-electron systems whose autoionization is inhibited by spin selection rules. They further suggest that such systems may also exist for higher electron number, and present possible experimental evidence of their detection. Although a more definite statement must await detailed calculations of the lifetimes expected for such states, we expect that the rapid increase with $Z$ of the spin-orbit mixing of doublet and quartet states will mean that analogous states for $Z=17$ will have lifetimes much shorter than nanoseconds and thus are probably not the states we observe. The consistency of the measured $K \alpha$ $\mathrm{x}$-ray energy with that expected from a two- or three-electron chlorine system, even though more than half of the associated chlorine ions appear to survive with four or more electrons, leads us to suspect that the states seen here may be better attributed to metastable two-electron systems which are accompanied by further electrons residing in shells of very high principal quantum number. Such a system would have to owe its metastability against Auger processes at least in part to the poor overlap of the wave function of the outer-shell electron with that of the inner shells, and to slow radiative progression from outer to inner shells by soft photon emission. Experimental observation of the radiation which should accompany such a progression and deduction of the overall excitation states of the outer shells of foil-excited ions would clearly be of importance in helping such an hypothesis to emerge from the speculation stage.

*Work partially supported by the U. S. Atomic Energy Commission under Contract No. AT(11-1)-2130.

${ }^{1}$ I. A. Sellin, D. J. Pegg, M. Brown, W. W. Smith, and B. Donally, Phys. Rev. Lett. 27, 1108 (1971).

${ }^{2}$ R. Marrus and R. W. Schmieder, Phys. Lett. $32 \mathrm{~A}$, 431 (1970); R. W. Schmieder and R. Marrus, Phys. Rev. Lett. 25, 1245 (1970); R. Marrus and R. W. Schmieder, Phys. Rev. Lett. 25, 1689 (1970); R. W. Schmieder and R. Marrus, Phys. Rev. Lett. 25, 1692 (1970); R. Marrus and R. W. Schmieder, Phys. Rev. A 5, 1160 (1972).

${ }^{3}$ L. L. House, Astrophys. J., Suppl. Ser. 18, 21 (1969).

\title{
Solution of the Dirac Equation for Strong External Fields*
}

\author{
Berndt Müller, Heinrich Peitz, Johann Rafelski, and Walter Greiner \\ Institut für Theoretische Physik der Universität Frankfurt, Frankfurt am Main, Germany \\ (Received 14 February 1972)

\begin{abstract}
The $1 s$ bound state of superheavy atoms and molecules reaches a binding energy of
\end{abstract} \\ $-2 m c^{2}$ at $Z \approx 169$. It is shown that the $K$ shell is still localized in $r$ space even beyond \\ this critical proton number and that it has a width $\Gamma$ (several keV large) which is a posi- \\ tron escape width for ionized $K$ shells. The suggestion is made that this effect can be ob- \\ served in the collision of very heavy ions (superheavy molecules) during the collision.
}

The discrete energy eigenvalues for an electron bound to a nucleus, which are obtained from the Dirac equation, lie between $m_{0} c^{2}$ and $-m_{0} c^{2}$, where $m_{0}$ is the electron's mass. The problem can be solved analytically in the case of a point nucleus; the energy eigenvalues are then given by the well-known Sommerfeld fine-structure formula. In this case the eigenvalues for the $1 s$ state become imaginary when the nuclear charge $Z$ becomes larger than 137. The problem may be cir- 
cumvented by attributing finite dimensions to the nucleus; still, the $1 s$ energy eigenvalue becomes equal to $-m_{0} c^{2}$, where the negative energy continuum begins, for a critical value, $Z_{\mathrm{cr}} \approx 169 .^{1}$ The exact value of $Z_{c r}$ depends on many assumptions concerning the potential in the vicinity of the nucleus. ${ }^{2}$ The interpretation of the theory has up to now been uncertain when the $1 s$ eigenvalue becomes equal to $-m_{0} c^{2}$. At this point the singleparticle theory seems to break down. Pieper and Greiner ${ }^{1}$ and later Popov $^{3}$ have interpreted this to mean that electron-positron pairs are created spontaneously.

It should be pointed out that this is not an academic problem, because even if superheavy elements cannot be readily produced, enough information could possibly be gathered in the collisions of heavy ions, such as $\mathrm{Pb}$ on $\mathrm{Pb}$ or $\mathrm{Cf}$ on $\mathrm{Cf}$, etc. ${ }^{2}$ to decide if electron-positron pairs are created spontaneously. In these collisions the adiabatic approximation should have some validity since the velocity of the electrons $v_{e} \approx c$ in the inner atomic orbitals is much greater then the relative nuclear motion $v_{\mathrm{i} \text { on }} \approx c / 20$. Thus during the heavy-ion collisions the energy eigenvalues of inner electrons will be steadily decreased (increasing binding), until a critical distance is reached where it becomes equal to $-m_{0} c^{2}$. Of course, the nuclei involved must have a minimum charge, say $Z=70-80$, so that the adiabaticity assumption is valid. It is also necessary that $Z_{1}+Z_{2}>Z_{\mathrm{cr}}$. When the critical separation between the two ions, $R_{\mathrm{cr}}$, is passed, it is expect$\mathrm{ed}^{1,3}$ that the $1 s$ eigenvalue "dives" into the nega- tive-energy continuum.

Our search for a reliable method of calculating this "diving" led us to consider the extent to which it is analogous to the process of autoionization as it was investigated by Fano ${ }^{4}$ in connection with ordinary atomic bound states imbedded in a continuum. Let $D$ be the free-particle Dirac operator. We then have to search for the bound states of the Hamiltonian $H=D+V(r, Z)$,

$$
H\left|\varphi_{n l}\right\rangle=E_{n l}\left|\varphi_{n l}\right\rangle,
$$

where $V(r, Z)=Z U(r)$ is the "rounded-off" Coulomb potential. Its essential $Z$ dependence has been factored out for convenience. $U(r)$ is only very weakly dependent on $Z$ via the extended nuclear charge distribution. Equation (1) can be solved as long as $Z \leqslant Z_{\mathrm{cr}}$. For $Z>Z_{\mathrm{cr}}$ we write $Z=Z_{\mathrm{cr}}+Z^{\prime}$; thus $V(r, Z)=Z_{\mathrm{cr}} U(r)+Z^{\prime} U(r)$. We consider now the $1 s$ bound-state eigenfunction $|\varphi\rangle$ for $Z=Z_{\mathrm{cr}}$. With $H_{0}=D+Z_{\mathrm{cr}} U(\gamma)$ we have

$$
H_{0}|\varphi\rangle=E_{0}|\varphi\rangle \approx-m_{0} c^{2}|\varphi\rangle \text {. }
$$

Further, let us consider the spherical $s$-wave functions of the negative-energy continuum, i.e., the eigenfunctions of (2) with $E<-m_{0} c^{2} ; H_{0}\left|\psi_{E}\right\rangle$ $=E\left|\psi_{E}\right\rangle$. We solve now the "diving problem" of the $1 s$ level of $H\left(Z_{\mathrm{cr}}+Z^{\prime}\right)$ in the basis of $H_{0}\left(Z_{\mathrm{cr}}\right)$. In doing this we neglect the small influence of the higher bound $s$ levels and of the positive $s$-wave continuum states (the next discrete states, $2 p_{1 / 2}$ and $2 s$, are separated by more than $200 \mathrm{keV}$ from the $1 s$ state at $Z=Z_{\mathrm{cr}}$ ). In other words, we diagonalize $H\left(Z_{\mathrm{cr}}+Z^{\prime}\right)$ of Eq. (1) in the restricted basis given by $|\varphi\rangle$ and by the negative continuum $\left|\psi_{E}\right\rangle$ and obtain

$$
\begin{aligned}
& \langle\varphi|H| \varphi\rangle=E_{0}+\Delta E_{0}, \quad \Delta E_{0}=\left\langle\varphi\left|Z^{\prime} U(r)\right| \varphi\right\rangle=Z^{\prime}\langle\varphi|U| \varphi\rangle, \\
& \left\langle\psi_{E^{\prime}}|H| \varphi\right\rangle=\left\langle\psi_{E^{\prime}}\left|H_{0}\right| \varphi\right\rangle+\left\langle\psi_{E^{\prime}}\left|Z^{\prime} U(r)\right| \varphi\right\rangle=Z^{\prime}\left\langle\psi_{E^{\prime}}|U| \varphi\right\rangle \equiv V_{E^{\prime}}, \\
& \left\langle\psi_{E^{\prime \prime}}|H| \psi_{E^{\prime}}\right\rangle=\left\langle\psi_{E^{\prime \prime}}\left|H_{0}\right| \psi_{E^{\prime}}\right\rangle+\left\langle\psi_{E^{\prime \prime}}\left|Z^{\prime} U\right| \psi_{E^{\prime}}\right\rangle=E^{\prime} \delta\left(E^{\prime \prime}-E^{\prime}\right)+U_{E^{\prime \prime} E^{\prime}} .
\end{aligned}
$$

The matrix elements $U_{E^{\prime \prime} E^{\prime}}$ describe the rearrangement of the continuum states under the additional potential $Z^{\prime} U(r)$. They can be neglected in our discussion here. ${ }^{5}$

The solution of (3) for $E<-m c^{2}$ has, within these approximations, the form

$$
\left|\Psi_{E}\right\rangle=a(E)|\varphi\rangle+\int_{-m_{0} c^{2}}^{-\infty} b_{E} \cdot \psi_{E^{\prime}} d E^{\prime}
$$

with the coefficients $a(E)$ and $b_{E}$ as determined in Ref. 4. The continuum functions $\left|\Psi_{E}\right\rangle$ are normalized in the usual way: $\left\langle\Psi_{\bar{E}} \mid \Psi_{E}\right\rangle=\delta(\bar{E}-E)$. We are mainly interested in $|a(E)|^{2}$, which is

$$
|a(E)|^{2}=\frac{\left|V_{E}\right|^{2}}{\left[E-\left(E_{0}+\Delta E_{0}\right)-F(E)\right]^{2}+\pi^{2}\left|V_{E}\right|^{4}}
$$

where

$$
F(E)=\mathrm{P} \int d E^{\prime}\left|V_{E}\right|^{2} /\left(E-E^{\prime}\right)
$$

and $\mathrm{P}$ indicates principal part. $|a(E)|^{2}$ has very strong resonance behavior if $\pi\left|V_{E}\right|^{2} \ll E_{0}+\Delta E_{0}$. We assume that $\pi\left|V_{E}\right|^{2}=\Gamma / 2$ is approximately constant. Then obviously $F(E) \approx 0$ and we have a 
Breit-Wigner resonance shape,

$$
|a(E)|^{2}=\frac{(2 \pi)^{-1} \Gamma}{\left[E-\left(E_{0}+\Delta E_{0}\right)\right]^{2}+\Gamma^{2} / 4} .
$$

Because we have chosen $|\varphi\rangle$ to be the bound $1 s$ eigenfunction for the critical charge, i.e., $E_{0}$ $\approx-m c^{2}, \Delta E \approx Z^{\prime}\langle\varphi|U(r)| \varphi\rangle=Z^{\prime} \delta$ obviously describes the energy shift due to the over-critical charge $Z^{\prime}$ of the bound state which has now become a resonance spread over the negative energy states $E$ with a width

$$
\Gamma=2 \pi\left|\left\langle\psi_{E}\left|Z^{\prime} U(r)\right| \varphi\right\rangle\right|^{2} \equiv Z^{\prime 2} \gamma
$$

We thus can learn the following facts: The bound level "dives" into the negative-energy continuum for $Z>Z_{\mathrm{cr}}$, proportional to $Z^{\prime}=Z-Z_{\mathrm{cr}}$. Then it obtains a width $\Gamma$ which is proportional to $Z^{\prime 2}$ $=\left(Z-Z_{\mathrm{cr}}\right)^{2}$. The resonance occurs at

$$
E=-m_{0} c^{2}-\left(Z-Z_{\mathrm{cr}}\right) \delta \text {. }
$$

The width $\Gamma$ is caused by the admixture of the negative-energy continuum states to the state $|\varphi\rangle$.

It can be easily seen that our formula corresponds closely to time-dependent perturbation theory. The decay probability per unit time is equal to $w=\Gamma / \hbar$, which gives with (8) Fermi's "golden rule" exactly for transition from the state $|\varphi\rangle$ to the continuum state $\left|\psi_{E}\right\rangle$ (the density of final states is equal to 1 , since the $\left|\psi_{E}\right\rangle$ are normalized to $\delta$ functions). According to this model, the state $|\varphi\rangle$ stays localized in $r$ space during the "diving-in" process. This means that the experimentally measured extension of the $K$-elec- tron cloud decreases (the cloud shrinks) as $Z$ increases, even beyond $Z_{\mathrm{cr}}$. On the other side, there will be a certain energy "delocalization" in the energy of the state $|\varphi\rangle$ as expressed by the width $\Gamma$. This width corresponds to a decaying state, if there is a nonvanishing final-state density. In the case that the $1 s$ state is not ionized, nothing happens; both electrons will, in a heavyion collision $\left(Z_{1}+Z_{2}>Z_{\mathrm{cr}}\right)$, dive into the negative energy sea after the critical distance $R_{\mathrm{cr}}$ is passed and emerge again when the heavy ions are again separated by more than $R_{\mathrm{cr}}$. To a fully occupied $1 s$ level there is no final state available. The width $\Gamma$ of the $K$ level in the negative-energy continuum (which is occupied by electrons according to Dirac) can in this case be understood in analogy to the spreading width of a doorway state. If on the other hand the $1 s$ state is ionized (once or twice), spontanous positron creation will take place. It is evident that energy is conserved in this process. The production of the electron-positron pair may be achieved without any additional energy, because the $1 s$ state is the final state for the produced electron, and the hole escapes and is interpreted, according to Dirac's hole theory as a positron with kinetic energy $T$ $=-E-m c^{2}{ }^{6}$

According to Eqs. (7) and (8) the probability $P(T) d T$ for creation of positrons with the kinetic energy $T$ is

$$
P(T) d T=\frac{(2 \pi)^{-1} Z^{\prime 2} \gamma d T}{\left[T-Z^{\prime} \delta\right]^{2}+Z^{\prime 4} \gamma^{2} / 4}
$$

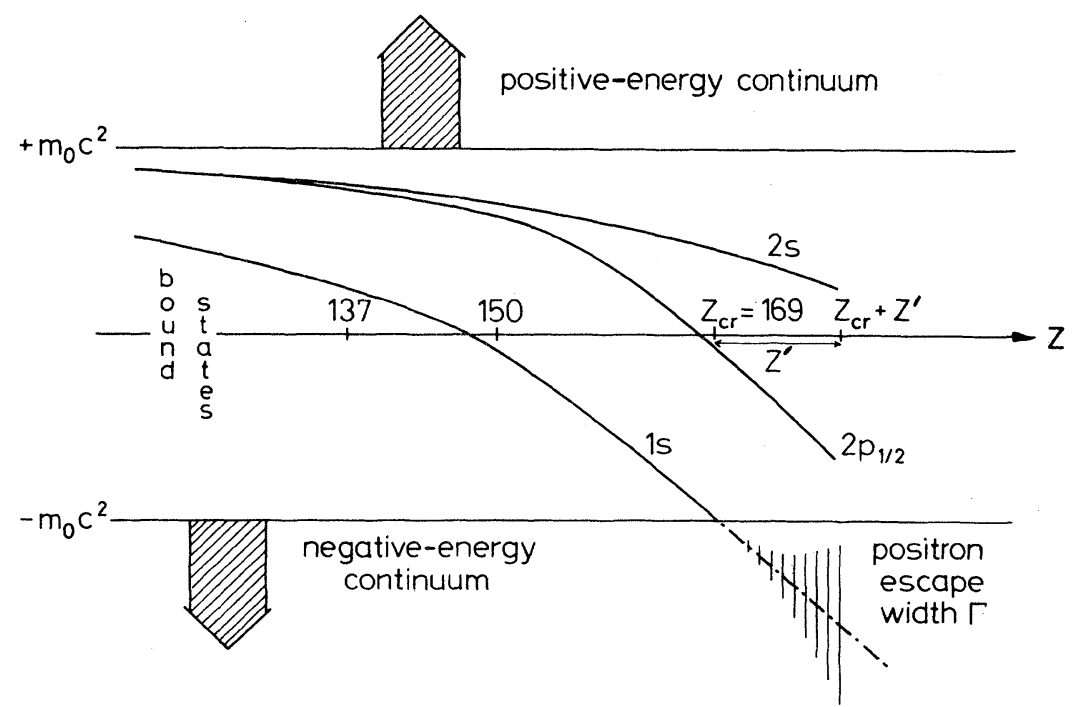

FIG. 1. Atomic energy levels in dependence of nuclear charge. The spreading of the positron escape width for the $1 s$ level is shown as a function of $Z^{\prime}$. 
which centers around $\Delta E=Z^{\prime} \delta$. The numerical estimate of $\delta$ and $\gamma$ gives $\delta \approx 30 \mathrm{keV}$ and $\gamma \approx 1.8 \mathrm{keV}$. This is illustrated in Fig. 1. Equation (10) may be easily integrated,

$$
W(T)=\int_{0}^{T} P\left(T^{\prime}\right) d T^{\prime}=\frac{1}{\pi}\left[\arctan \left(\frac{2(T-\Delta E)}{\Gamma}\right)-\arctan \left(\frac{-2 \Delta E_{0}}{\Gamma}\right)\right] ;
$$

if $2 \Delta E>\Gamma$ or $2 \delta>Z^{\prime} \gamma$, we obviously have $W(\infty)$ $\approx 1$. This means that in this case a sharp resonance occurs in $P(T)$ for $T=Z^{\prime} \delta$; most positrons are created with this kinetic resonance energy. ${ }^{7}$ Furthermore the charge density of the shrinking $K$ shell, i.e., $\bar{\varphi} \varphi(r) \int_{-m_{0} c^{2}}^{-\infty} a^{*}(E) a(E) d E$, changes continuously in the "diving" process. This means the following: According to the wave function (4), which describes the mixture of the bound state $\varphi$ with the continuum $\psi_{E}$, the latter is "deformed" by the former. The probability for finding the state $\varphi$ is $\int_{-m_{0} c^{2}}^{-\infty} a *(E) a(E) d E \approx 1$. Therefore, the charge distribution of the $K$ shell is still localized also, even though spread out in energy (width $\Gamma$ ) after the "diving" process. In the opposite case, $\Delta E<\Gamma$ or $2 \delta<Z^{\prime} \gamma$, the results become more complex, since the assumptions that $\Gamma$ is energy independent and $F(E) \approx 0$ do not hold. One can expect, however, that the kinetic-energy spectrum of the positrons becomes rather broad. Note that in the latter case the present model should also be improved by incorporating the threshold behavior of the $1 s$ state as it passes through $-m_{0} c^{2}$; in that case the continuum states are strongly coupled to the $1 s$ state even for $E$ $>-m_{0} c^{2}$.

We remark finally that these considerations are based on the assumption that effects such as nonlinearities in the Dirac equation, etc., which could prevent the "diving" process, are not present. However, for nonlinear electrodynamic effects as discussed recently, ${ }^{2}$ the conclusions reached in this note are valid. In this case only the critical charge $Z_{\mathrm{cr}}$ is shifted towards higher values. It is also assumed that quantum-electro- dynamic effects (vacuum polarization, etc.) do not become comparable to the "diving" effects discussed here. Because the half-life of the $K$ hole in the negative-energy continuum is about $\tau=\hbar / \Gamma=\hbar / Z^{\prime 2} \gamma$, the ions in the experiment must be chosen such that $Z^{\prime}$ is large, so that $\tau$ is small compared to the collision time (large positron escape width). The escaping positrons will then also have rather high kinetic energy (see Fig. 1).

We acknowledge interesting discussions with J. H. D. Jensen (Heidelberg).

*Work supported by the Deutsche Forschungsgemeinschaft, by the Budesministerium für Bildung und Wissenschaft, and by Gesellschaft für Schwerionen-Forschung.

${ }^{1}$ W. Pieper and W. Greiner, Z. Phys. 218, 327 (1969); see also H. G. Dosch, J. H. D. Jensen, and V. F. Müller, "Einige Bemerkungsen zum Kleinschen Paradoxon" (to be published).

${ }^{2}$ J. Rafelski, L. P. Fulcher, and W. Greiner, Phys. Rev. Lett. 27, 958 (1971).

${ }^{3}$ V. S. Popov, Zh. Eksp. Teor. Fiz. 59, 965 (1970)

[Sov. Phys. JETP 32, 526 (1971)].

${ }^{4}$ U. Fano, Phys. Rev. 124, 1866 (1961).

${ }^{5}$ Otherwise $U_{E^{\prime \prime} E^{\prime}}$ may be prediagonalized.

${ }^{6}$ This can also be expressed by stating that the oneelectron configuration $a_{1 s} \uparrow^{\dagger}|0\rangle$ is degenerate with the two-electron, one-positron congigurations $a_{1 s} \uparrow^{\dagger} a_{1 s} t^{\dagger}$ $\times \beta_{s E}|0\rangle$, where $E$ is continuous.

${ }^{7}$ In a concrete ion-ion scattering experiment the total kinetic-energy distribution of escaping positrons will be obtained by folding (11) with the probability for reaching an effective $Z^{\prime}$ in the collision. 